\title{
Heat and Mass Transfer Effects on Unsteady MHD Free Convection Flow Past a Vertical Permeable Moving Plate with Radiation
}

\author{
M.C. Krishna Reddy ${ }^{1}$, G. Murali ${ }^{2}$, S. Sivaiah ${ }^{3}$, NVN Babu $^{2}$ \\ ${ }^{1}$ Department of Mathematics, OsmaniaUniversity, Hyderabad India \\ ${ }^{2}$ Department of Mathematics, GITAM University, Rudraram,India \\ ${ }^{3}$ Department of Mathematics, BVRIT,Medak,India \\ gundagani20@yahoo.com
}

\begin{abstract}
In this analysis, the effects of radiation have been discussed on unsteady MHD free convection heat and mass transfer flow on a viscous, incompressible, electrically conducting fluid past a vertical permeable moving plate with radiation. The non-linear partial differential equations governing the flow have been solved numerically using finite element method. Graphical results for velocity, temperature and concentration profiles have been obtained, to show the effects of different parameters entering in the problem. Such flow problems are important in many processes, in which there is combined heat and mass transfer with radiation. It has been observed that the velocity increase with the increase in the radiation parameter and there is a increase in temperature with the increase in the value of radiation parameter.
\end{abstract}

Keywords: Heat and Mass transfer, MHD, Radiation, FEM

\section{Introduction}

The effect of radiation on MHD flow and heat transfer problem have become more important industrially. At high operating temperature, radiation effect can be quite significant. Many processes in engineering areas occur at high temperature and a knowledge of radiation heat transfer becomes very important for the design of the pertinent equipment. Nuclear power plants, gas turbines and the various propulsion devices for aircraft, missiles, satellites and space vehicles are examples of such engineering areas. Bestman [1] examined the natural convection boundary 
layer with suction and mass transfer in a porous medium. His results confirmed the hypothesis that suction stabilises the boundary layer and affords the most efficient method in boundary layer control yet known. Abdus Sattar and Hamid Kalim [2] investigated the unsteady free convection interaction with thermal radiation in a boundary layer flow past a vertical porous plate. Makinde [3] examined the transient free convection interaction with thermal radiation of an absorbing-emitting fluid along moving vertical permeable plate. Recently, Ibrihem et al. [4] have studied nonclassical thermal effects in Stokes' second problem for micropolar fluids by used perturbation method.

Muthucumaraswamy and Ganesan [5] studied effect of the chemical reaction and injection on flow characteristics in an unsteady upward motion of an isothermal plate. Deka et al. [6] studied the effect of the first-order homogeneous chemical reaction on the process of an unsteady flow past an infinite vertical plate with a constant heat and mass transfer. Chamkha [7] studied the MHD flow of a numerical of uniformly stretched vertical permeable surface in the presence of heat generation/absorption and a chemical reaction. Soundalgekar and Patti [8] studied the problem of the flow past an impulsively started isothermal infinite vertical plate with mass transfer effects. The effect of foreign mass on the freeconvection flow past a semi-infinite vertical plate was studied [9]. Chamkha [10] assumed that the plate is embedded in a uniform porous medium and moves with a constant velocity in the flow direction in the presence of a transverse magnetic field. Raptis [11] investigate the steady flow of a viscous fluid through a very porous medium bounded by a porous plate subjected to a constant suction velocity by the presence of thermal radiation. Raptis and Perdikis [12] studied the unsteady free convection flow of water near $40 \mathrm{C}$ in the laminar boundary layer over a vertical moving porous plate.

Chambre et al [13] have analyzed a first order chemical reaction in the neighborhood of a stationary horizontal plate. Das et al [14] have studied the effect of homogeneous first order chemical reaction on the flow past an impulsively started infinite vertical plate with uniform heat flux and mass transfer. Again, mass transfer effects on moving isothermal vertical plate in the presence of chemical reaction studied by Das et al [15]. The dimensionless governing equations were solved by the usual Laplace-trans form technique and the solutions are valid only at lower time level. Radiation and chemical reaction effects on isothermal vertical oscillating plate with variable mass diffusion has been studied by Manivannan et al [16].

In spite of all these studies, the heat and mass transfer effects on unsteady magnetohydrodynamic free convection flow past a vertical permeable moving plate with radiation. The problem is governed by the system of non-linear partial differential equations solved numerically by using Galerkin finite element method. Which is more economical from computational point of view? 


\section{Mathematical analysis}

Consider unsteady two-dimensional flow of a laminar, viscous, electrically conducting and heat-absorbing fluid past a semi-infinite vertical permeable moving plate embedded in a uniform porous medium and subjected to a uniform transverse magnetic field in the presence of thermal and concentration buoyancy effects. It is assumed that there is no applied voltage which implies the absence of an electrical field. The fluid properties are assumed to be constant except that the influence of density variation with temperature has been considered only in the body-force term. The concentration of diffusing species is very small in comparison to other chemical species, the concentration of species far from the wall, $\mathrm{C} \infty$, is infinitesimally small [5] and hence the Soret and Dufour effects are neglected. The chemical reactions are taking place in the flow and all thermophysical properties are assumed to be constant of the linear momentum equation which is approximated according to the Boussinesq approximation. Due to the semi-infinite plane surface assumption, the flow variables are functions of $\mathrm{y}^{*}$ and the time $\mathrm{t}^{*}$ only. Under these assumptions, the equations that describe the physical situation are given by

$$
\begin{gathered}
\frac{\partial v^{*}}{\partial y^{*}}=0, \\
\frac{\partial u^{*}}{\partial t^{*}}+v^{*} \frac{\partial u^{*}}{\partial y^{*}}=v \frac{\partial^{2} u^{*}}{\partial y^{* 2}}-v \frac{u^{*}}{K^{\prime}}-\frac{\sigma B_{0}^{2} u^{*}}{\rho}+g \beta_{T}\left(T^{*}-T_{\infty}\right)+g \beta_{C}\left(C^{*}-C_{\infty}\right), \\
\frac{\partial T^{*}}{\partial t^{*}}+v^{*} \frac{\partial T^{*}}{\partial y^{*}}=\frac{k}{\rho c_{p}} \frac{\partial^{2} T^{*}}{\partial y^{* 2}}-\frac{1}{\rho c_{p}}\left(\frac{\partial q_{r}}{\partial y^{\prime}}\right) \\
\frac{\partial C^{*}}{\partial t^{*}}+v^{*} \frac{\partial C^{*}}{\partial y^{*}}=D \frac{\partial^{2} C^{*}}{\partial y^{* 2}}-K_{r}^{*} C^{*}
\end{gathered}
$$

where $x^{*}, y^{*}$, and $t^{*}$ are the dimensional distances along and perpendicular to the plate and dimensional time, respectively. $\mathrm{u}^{*}$ and $\mathrm{v}^{*}$ are the components of dimensional velocities along $x^{*}$ and $y^{*}$ directions, respectively, $\mathrm{T}^{*}$ is the dimensional temperature, $\mathrm{C}^{*}$ is the dimensional concentration, $\mathrm{Cw}$ and $\mathrm{Tw}$ are the concentration and temperature at the wall, respectively. $\mathrm{C} \infty$ and $\mathrm{T} \infty$ are the free stream dimensional concentration and temperature, respectively. ${ }^{\rho}$ is the fluid density, $\mathrm{v}$ is the kinematic viscosity, $\mathrm{cp}$ is the specific heat at constant pressure, ${ }^{\sigma}$ is the fluid electrical conductivity, Bo is the magnetic induction, $\mathrm{K}^{*}$ is the 
permeability of the porous medium, $Q_{I}^{*}$ is the coefficient of proportionality for the absorption of radiation, $\mathrm{D}$ is the mass diffusivity, $\mathrm{g}$ is the gravitational acceleration, and $\beta_{T}$ and $\beta_{c}$ are the thermal and concentration expansion coefficients, respectively and $\mathrm{Kl}$ is the chemical reaction parameter. The magnetic and viscous dissipations are neglected in this study. The third and fourth terms on the RHS of the momentum equation (2) denote the thermal and concentration buoyancy effects, respectively. Also, the second and third terms on the RHS of the energy equation (3) represents the heat and radiation absorption effects, respectively. It is assumed that the permeable plate moves with a variable velocity in the direction of fluid flow. In addition, it is assumed that the temperature and the concentration at the wall as well as the suction velocity are exponentially varying with time.

Under these assumptions, the appropriate boundary conditions for the velocity, temperature and concentration fields are

$$
\left.\begin{array}{l}
u^{*}=u_{P}^{*}, T^{*}=T_{w}+\varepsilon\left(T_{w}-T_{\infty}\right) e^{n^{*} t^{*}}, C^{*}=C_{w}+\varepsilon\left(C_{w}-C_{\infty}\right) e^{n^{*} t^{*}}, y^{*}=0, \\
u^{*}=0, T^{*} \rightarrow T_{\infty}, C^{*} \rightarrow C_{\infty}, y^{*} \rightarrow \infty
\end{array}\right\}
$$

where $u_{p}^{*}$ is the wall dimensional velocity, $\mathrm{n}^{*}$ is constant. It is clear from Eq. (1) that the suction velocity at the plate surface is a function of time only. Assuming that it takes the following exponential form:

$$
\mathrm{V}^{*}=-\mathrm{V} 0\left(1+\varepsilon \operatorname{Aen}^{*} \mathrm{t}^{*}\right)
$$

where $\mathrm{A}$ is a real positive constant, $\varepsilon$ and $\varepsilon \mathrm{A}$ are small less than unity, and Vo is a scale of suction velocity which has non-zero positive constant. Introducing the dimensionless quantities:

$$
\begin{aligned}
& u=\frac{u^{*}}{V_{0}}, v=\frac{v^{*}}{V_{0}}, y=\frac{V_{0} y^{*}}{v}, t=\frac{V_{0}^{2} t^{*}}{v}, u_{P}=\frac{u_{P}^{*}}{V_{0}}, n=\frac{n^{*} v}{V_{0}^{2}}, \quad \theta=\frac{T^{*}-T_{\infty}}{T_{w}-T_{\infty}}, C=\frac{C^{*}-C}{C_{w}-C_{\infty}} \\
& G r=\frac{v g \beta\left(T_{w}-T_{\infty}\right)}{V_{0}^{3}}, G m=\frac{v g \beta_{C}\left(C_{w}-C_{\infty}\right)}{V_{0}^{3}}, \operatorname{Pr}=\frac{\mu c_{p}}{k}, \quad M^{2}=\frac{\sigma \beta_{0}^{2} v}{\rho V_{0}^{2}}, \\
& K_{0}=\frac{K^{*} V_{0}^{2}}{v^{2}}, S_{C}=\frac{v}{D}, \quad R=\frac{16 \sigma_{s} T_{\infty}^{3}}{3 K_{e} k}, K_{r}=\frac{K_{r}^{*} \nu}{V_{0}^{2}},
\end{aligned}
$$

In view of the above non-dimensional variables, the basic field Eqs. (2)-(4) can be expressed in non dimensional form as 


$$
\begin{aligned}
& \frac{\partial u}{\partial t}-\left(1+\varepsilon A e^{n t}\right) \frac{\partial u}{\partial y}=G r \theta+G m C+\frac{\partial^{2} u}{\partial y^{2}}-\frac{u}{K_{o}\left(1+\varepsilon A e^{n t}\right)}-M^{2} u \\
& \frac{\partial \theta}{\partial t}-\left(1+\varepsilon A e^{n t}\right) \frac{\partial \theta}{\partial y}=\frac{1}{\operatorname{Pr}} \frac{\partial^{2} \theta}{\partial y^{2}}-R \theta \\
& \frac{\partial C}{\partial t}-\left(1+\varepsilon A e^{n t}\right) \frac{\partial C}{\partial y}=\frac{1}{S c} \frac{\partial^{2} C}{\partial y^{2}}-K_{r} C
\end{aligned}
$$

The corresponding boundary conditions are

$$
\begin{gathered}
u=u_{p} \theta=1+\varepsilon e^{n t}, \quad C=1+\varepsilon e^{n t}, \quad \text { on } y=0 \\
u \rightarrow 0, \theta \rightarrow 0, C \rightarrow 0 \quad \text { as } y \rightarrow \infty,
\end{gathered}
$$

where $G r, G m, M, K_{o}, \operatorname{Pr}, R$ and $S c$ are the thermal Grashof number, solutal Grashof number, magnetic field parameter, permeability parameter, Prandtl number, radiation parameter and Schmidt number respectively.

The mathematical statement of the problem is now complete and embodies the solution of Eqs.(8)-(10) subject to boundary condition(11).

\section{Method of Solution}

By applying Galerkin finite element method for equation (8) over the element $(e),\left(y_{j} \leq y \leq y_{k}\right)$ is:

$$
\int_{y_{j}}^{y_{k}} N^{(e)^{T}}\left[\frac{\partial^{2} u^{(e)}}{\partial y^{2}}+P \frac{\partial u^{(e)}}{\partial y}-\frac{\partial u^{(e)}}{\partial t}-M_{1} u^{(e)}+R_{1}\right] d y=0
$$

Where $P=1+\varepsilon A e^{n t}, \quad M_{1}=M^{2}+\frac{1}{K_{o} P}, \quad R_{1}=G r \theta+G m \phi$

Integrating the first term in equation (12) by parts one obtains

$$
\left.N^{(e)^{T}} \frac{\partial u^{(e)}}{\partial y}\right\}_{y_{j}}^{y_{k}}-\int_{y_{j}}^{y_{k}}\left\{\frac{\partial N^{(e)^{T}}}{\partial y} \frac{\partial u^{(e)}}{\partial y}-N^{(e)^{T}}\left(P \frac{\partial u^{(e)}}{\partial y}+\frac{\partial u^{(e)}}{\partial t}+M_{1} u^{(e)}-R_{1}\right)\right\} d y=0
$$

Neglecting the first term in equation (13),then 
$\int_{y_{j}}^{y_{k}}\left\{\frac{\partial N^{(e)^{T}}}{\partial y} \frac{\partial u^{(e)}}{\partial y}-N^{(e)^{T}}\left(P \frac{\partial u^{(e)}}{\partial y}-\frac{\partial u^{(e)}}{\partial t}-M_{1} u^{(e)}+R_{1}\right)\right\} d y=0$

Let $u^{(e)}=N^{(e)} \phi^{(e)}$ be the linear piecewise approximation solution over the element $(e),\left[y_{j} \leq y \leq y_{k}\right]$, where $N^{(e)}=\left[\begin{array}{ll}N_{j} & N_{k}\end{array}\right], \quad \phi^{(e)}=\left[\begin{array}{ll}u_{j} & u_{k}\end{array}\right]^{T}$ and $N_{j}=\frac{y_{k}-y}{y_{k}-y_{j}}, \quad N_{k}=\frac{y-y_{j}}{y_{k}-y_{j}}$ are the basis functions. One obtains:

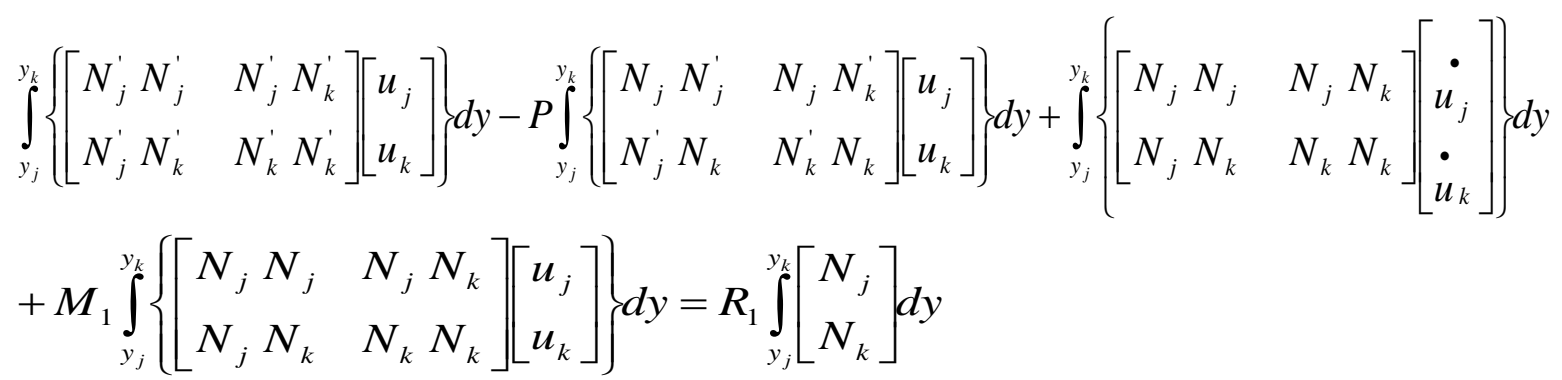

Simplifying we get

$\frac{1}{l^{(e)^{2}}}\left[\begin{array}{cc}1 & -1 \\ -1 & 1\end{array}\right]\left[\begin{array}{l}u_{j} \\ u_{k}\end{array}\right]-\frac{P}{2 l^{(e)}}\left[\begin{array}{ll}-1 & 1 \\ -1 & 1\end{array}\right]\left[\begin{array}{l}u_{j} \\ u_{k}\end{array}\right]+\frac{1}{6}\left[\begin{array}{cc}2 & 1 \\ 1 & 2\end{array}\right]\left[\begin{array}{l}u_{j}^{\cdot} \\ u_{k}^{\bullet}\end{array}\right]+\frac{M_{1}}{6}\left[\begin{array}{ll}2 & 1 \\ 1 & 2\end{array}\right]\left[\begin{array}{l}u_{j} \\ u_{k}\end{array}\right]=\frac{R_{1}}{2}\left[\begin{array}{l}1 \\ 1\end{array}\right]$

where prime and dot denotes differentiation w.r.t $y$ and time $t$ respectively. Assembling the element equations for two consecutive elements $y_{i-1} \leq y \leq y_{i}$ and $y_{i} \leq y \leq y_{i+1}$ following is obtained:

$$
\begin{aligned}
& \frac{1}{l^{(e)^{2}}}\left[\begin{array}{rrr}
1 & -1 & 0 \\
-1 & 2 & -1 \\
0 & -1 & 1
\end{array}\right]\left[\begin{array}{l}
u_{i-1} \\
u_{i} \\
u_{i+1}
\end{array}\right]-\frac{P}{2 l^{(e)}}\left[\begin{array}{rrr}
-1 & 1 & 0 \\
-1 & 0 & 1 \\
0 & -1 & 1
\end{array}\right]\left[\begin{array}{l}
u_{i-1} \\
u_{i} \\
u_{i+1}
\end{array}\right]+\frac{1}{6}\left[\begin{array}{lll}
2 & 1 & 0 \\
1 & 4 & 1 \\
0 & 1 & 2
\end{array}\right]\left[\begin{array}{l}
\cdot \\
u_{i-1} \\
\dot{u}_{i} \\
u_{i+1}
\end{array}\right] \\
& +\frac{M_{1}}{6}\left[\begin{array}{rrr}
2 & 1 & 0 \\
1 & 4 & 1 \\
0 & 1 & 2
\end{array}\right]\left[\begin{array}{l}
u_{i-1} \\
u_{i} \\
u_{i+1}
\end{array}\right]=\frac{R_{1}}{2}\left[\begin{array}{l}
1 \\
2 \\
1
\end{array}\right]
\end{aligned}
$$


Now put row corresponding to the node $i$ to zero, from equation (14) the difference schemes with $l^{(e)}=h$ is:

$$
\frac{1}{h^{2}}\left[-u_{i-1}+2 u_{i}-u_{i+1}\right]-\frac{P}{2 h}\left[-u_{i-1}+u_{i+1}\right]+\frac{1}{6}\left[\dot{u_{i-1}}+4 \dot{u_{i}}+\dot{u}_{i+1}\right]+\frac{M_{1}}{6}\left[u_{i-1}+4 u_{i}+u_{i+1}\right]=R_{1}
$$

Applying Crank-Nicholson method to the above equation then we gets

$$
A_{1} u_{i-1}^{j+1}+A_{2} u_{i}^{j+1}+A_{3} u_{i+1}^{j+1}=A_{4} u_{i-1}^{j}+A_{5} u_{i}^{j}+A_{6} u_{i+1}^{j}+P^{*}
$$

Where $A_{1}=2-6 r+3 P h r+M_{1} k, \quad A_{2}=8+12 r+4 M_{1} k$,

$$
\begin{aligned}
& A_{3}=2-6 r-3 P h r+M_{1} k, \quad A_{4}=2+6 r-3 P h r-M_{1} k, \\
& A_{5}=8-12 r-4 M_{1} k, \quad A_{6}=2+6 r+3 P h r-M_{1} k, \\
& P^{*}=12(G r) k \theta_{i}^{j}+12(G m) k C_{i}^{j} ;
\end{aligned}
$$

Applying similar procedure to equation (9) and (10) then we gets

$$
\begin{aligned}
& B_{1} \theta_{i-1}^{j+1}+B_{2} \theta_{i}^{j+1}+B_{3} \theta_{i+1}^{j+1}=B_{4} \theta_{i-1}^{j}+B_{5} \theta_{i}^{j}+B_{6} \theta_{i+1}^{j}+P^{* *} \\
& C_{1} C_{i-1}^{j+1}+C_{2} C_{i}^{j+1}+C_{3} C_{i+1}^{j+1}=C_{4} C_{i-1}^{j}+C_{5} C_{i}^{j}+C_{6} C_{i+1}^{j}
\end{aligned}
$$

Where $B_{1}=2 \operatorname{Pr}-6 r+3 \operatorname{PPr} h r+\operatorname{Pr} R k, \quad B_{2}=8 \operatorname{Pr}+12 r+4 \operatorname{Pr} R k$,

$$
\begin{array}{rlr}
B_{3}=2 \operatorname{Pr}-6 r-3 P \operatorname{Pr} h r+\operatorname{Pr} R k, & B_{4}=2 \operatorname{Pr}+6 r-3 P \operatorname{Pr} h r-\operatorname{Pr} R k, \\
B_{5}=8 \operatorname{Pr}-12 r-4 \operatorname{Pr} R k, & \\
B_{6}=2 \operatorname{Pr}+6 r+3 P \operatorname{Pr} h r-\operatorname{Pr} R k, & C_{1}=2 S c-6 r+3 P S c r h+S c K_{r} k, \\
C_{2}=8 S c+12 r+4 S c K_{r} k, & \\
C_{3}=2 S c-6 r-3 P S c r h+S c K_{r} k, & C_{4}=2 S c+6 r-3 P S c r h-S c K_{r} k, \\
C_{5}=8 S c-12 r-4 S c K_{r} k, & C_{6}=2 S c+6 r+3 P S c r h-S c K_{r} k,
\end{array}
$$

Here $r=\frac{k}{h^{2}}$ and $h, k$ are the mesh sizes along $y$-direction and time $t$-direction respectively. Index $i$ refers to the space and $j$ refers to the time. In 
equations (16) - (18), taking $i=1(1) n$ and using initial and boundary conditions (11), the following system of equations are obtained:

$A_{i} X_{i}=B_{i} \quad i=1(1) 3$

Where $A_{i}$ 's are matrices of order $n$ and $X_{i}, B_{i}$ 's column matrices having $n$-components. The solutions of above system of equations are obtained by using Thomas algorithm for velocity, temperature and concentration. Also, numerical solutions for these equations are obtained by C-programme. In order to prove the convergence and stability of Galerkin finite element method, the same C-programme was run with slightly changed values of $h$ and $k$ and no significant change was observed in the values of $u, \theta$ and $C$. Hence, the Galerkin finite element method is stable and convergent.

\section{Results and Discussion}

Numerical evaluation of the analytical results reported in the previous section was performed and a representative set of results is reported graphically in figs.1-12. These results are obtained to illustrate the influence of the thermal Grashof number $G r$, Solutal Grashof number $G m$, magnetic parameter $M$, Permeability parameter $K$, thermal radiation $R$, Prandtl number $\operatorname{Pr}$, Schmidt number $S c$ and chemical reaction parameter $K_{r}$ on the velocity, temperature and the concentration profiles, while the values of the physical parameters are fixed at real constants

With

$$
\begin{aligned}
& G r=G m=2.0, M=0.2, K=0.5, \operatorname{Pr}=0.71, R=1.0, S c=0.6, K_{r}=0.5, A=0.5, u_{p}=0.5, \\
& \varepsilon=0.2, n=0.1, t=1.0 \text { All graphs therefore correspond to these values unless } \\
& \text { specifically indicated on the appropriate graph. }
\end{aligned}
$$

Fig 1 presents typical velocity profiles in the boundary layer for various values of the thermal Grashof number $G r$, while all other parameters are kept at some fixed values. The thermal Grashof number $G r$ defines the ratio of the species buoyancy force to the viscous hydrodynamic force. As expected, the fluid velocity increases and the peak value is more distinctive due to increase in the species buoyancy force. The velocity distribution attains a distinctive maximum value in 
the vicinity of the plate and then decreases properly to approach the free stream value.

The influence of the Solutal Grashof number $G m$ on the velocity is presented in Fig 2. The Solutal Grashof number signifies the relative effect of the thermal buoyancy force to the viscous hydrodynamic force in the boundary layer. As expected, it is observed that there is a rise in the velocity due to the enhancement of thermal buoyancy force. Here, the positive values of $G m$ correspond to cooling of the plate. Also, as $G m$ increases, the peak values of the velocity increases rapidly near the porous plate and then decays smoothly to the free stream velocity.

For different values of the magnetic field parameter $\mathrm{M}$, the velocity profile are plotted in Fig. 3. It is obvious that the effect of increasing values of the magnetic field parameter results in a decreasing velocity distribution across the boundary layer. Fig. 4 illustrate the variation of velocity distribution across the boundary layer for various values of the permeability parameter $\mathrm{K}$. The velocity increases with a increase in permeability parameter $\mathrm{K}$.

Figs 5 and 6 illustrate the velocity and temperature profiles for different values of the Prandtl number Pr. The Prandtl number defines the ratio of momentum diffusivity to thermal diffusivity. The numerical results show that the effect of increasing values of Prandtl number results in a decreasing velocity (Fig 5). From Fig 6, it is observed that an increase in the Prandtl number results a decrease of the thermal boundary layer thickness and in general lower average temperature within the boundary layer. The reason is that smaller values of $\operatorname{Pr}$ are equivalent to increasing the thermal conductivities, and therefore heat is able to diffuse away from the heated plate more rapidly than for higher values of $\operatorname{Pr}$. Hence in the case of smaller Prandtl numbers as the boundary layer is thicker and the rate of heat transfer is reduced.

For different values of the radiation parameter $R$ the velocity and temperature profiles are plotted in Figs.7 and 8. It is obvious that an increase in the radiation parameter $R$ results an increasing in the velocity and temperature profiles within the boundary layer, as well as an increasing in the momentum and thermal thickness. This is because the large $R$ values correspond to an increased dominance of conduction over radiation thereby increasing buoyancy force (thus, vertical velocity) and thickness of the thermal and momentum boundary layers. 
Figs.9 and 10 display the effects of the Schmidt number $S c$ on the velocity, temperature and concentration profiles, respectively. As the Schmidt number increases, the concentration decreases. This causes the concentration buoyancy effects to decrease yielding a reduction in the fluid velocity. The reduction in the velocity, temperature and concentration profiles are accompanied by simultaneous reductions in the momentum and concentration boundary layers thickens. These behaviors are clearly shown in Figs. 9 and 10.

Figs. 11 and 12, displays results for the velocity and concentration distributions respectively. It is seen, that the velocity and concentration increases with decreasing the chemical reaction parameter $K_{r}$.Also, we observe that the magnitude of the stream wise velocity increases and the inflection point for the velocity distribution moves further away from the surface.

\section{Concluding Remarks}

The plate velocity was maintained at a constant value and the flow was subjected to a transverse magnetic field. The resulting partial differential equations were transformed into a set of ordinary differential equations using finite element method. Numerical results were performed and some graphical results were obtained to illustrate the details of the flow and heat and mass transfer characteristic and their dependence on some of the physical parameters. It was found that the velocity profiles increased due to decrease in chemical reaction parameter, the Schmidt number, magnetic field and Prandtl number parameters while it increased due to increases in thermal Grashof number, Solutal Grashof number, radiation parameter and Permeability parameters. However, an increase temperature profile is a function of an increase in radiation parameter while it decreased due to increases in Prandtl number. Also, it was found that the concentration profile increased due to decreases in the chemical reaction parameter and the Schmidt number.

\section{References}

[1] Bestman AR. Natural convection boundary layer with suction and mass transfer in a porous Medium. Int J Energy Res 1990;14:389-96.

[2] Abdus Sattar MD, Hamid Kalim MD. Unsteady free-convection interaction with thermal Radiation in a boundary layer flow past a vertical porous plate. J Math Phys Sci 1996;30:25-37. 
[3] Makinde OD. Free convection flow with thermal radiation and mass transfer past a moving Vertical plate. Int Comm Heat Mass Transfer 2005;32:1411-9.

[4] Ibrihem FS, Hassanien IA, Bakr AA. Nonclassical thermal effects in stokes' second problem For micropolar fluids. ASME J Appl Mech 2005;72:468-74.

[5] Muthucumaraswamy R, Ganesan P. Effect of the chemical reaction and injection on flow Characteristics in an unsteady upward motion of an isothermal plate. J Appl Mech Tech Phys 2001;42:665-71.

[6] Deka R, Das UN, Soundalgekar VM. Effect of mass transfer on flow past an impulsively Started infinite vertical plate with constant heat flux and chemical reaction. Forschung im Ingenieurwesen 1994;60:284-7.

[7] Chamkha AJ. MHD flow of a numerical of uniformly stretched vertical permeable surface in The presence of heat generation/absorption and a chemical reaction. Int Comm Heat Mass Trasfer 2003:30:413-22.

[8] Soundalgekar VM, Patti MR. Stokes problem for a vertical plate with constant heat flux.Astrophys Space Sci1980;70:179-82.

[9] Gebhart B, Pera L. The nature of vertical natural convection flow resulting from the Combined buoyancy effects of thermal and mass diffusion. J Heat Mass Transfer 1971;14:2025-50.

[10] Chamkha AJ. Unsteady MHD convective heat and mass transfer past a semiinfinite vertical permeable moving plate with heat absorption. Int J Eng Sci 2004;42:217-30.

[11] Raptis A. Radiation and free convection flow through a porous medium. Int Comm Heat Mass Transfer 1998;25:289-95.

[12] Raptis A, Perdikis C. Free convection flow of water near 4oC past a moving plate.Forschung im Ingenieurwesen 2002;67:206-8.

[13] Chambre PL, Young JD (1958). On the diffusion of a chemically reactive species in a laminar boundary layer flow, The Physics of Fluids, 1(1), pp. 4854.

[14] Das UN, Deka RK, Soundalgekar VM (1999). Effects of mass transfer on flow past an impulsively started infinite vertical plate with chemical reaction, The Bulletin, GUMA, 5(1), pp. 13-20.

[15] Das UN, Deka RK, Soundalgekar VM (1994). Effects of mass transfer on flow past an impul sivelystarted in fi nite ver ti cal plate with constant heat flux and chemial reaction, Forschung imIngenieurwesen, 60(10), pp. 284287.

[16] Manivannan K, Muthucumaraswamy R, Venu T (2009). Radiation and chemical reaction effects on isothermal vertical oscillating plate with variable mass diffusion, Thermal Science., 13(2), pp. 155-162. 


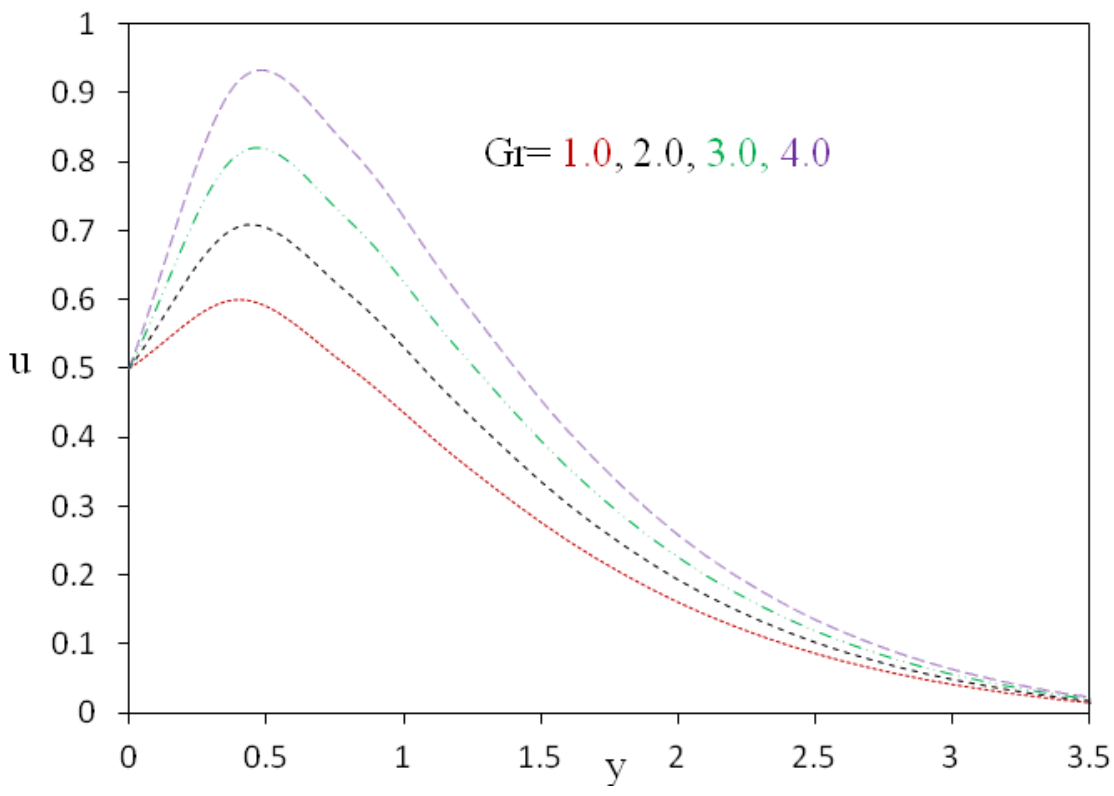

Fig.1. Velocity profiles against $y$ for different values of $G r$

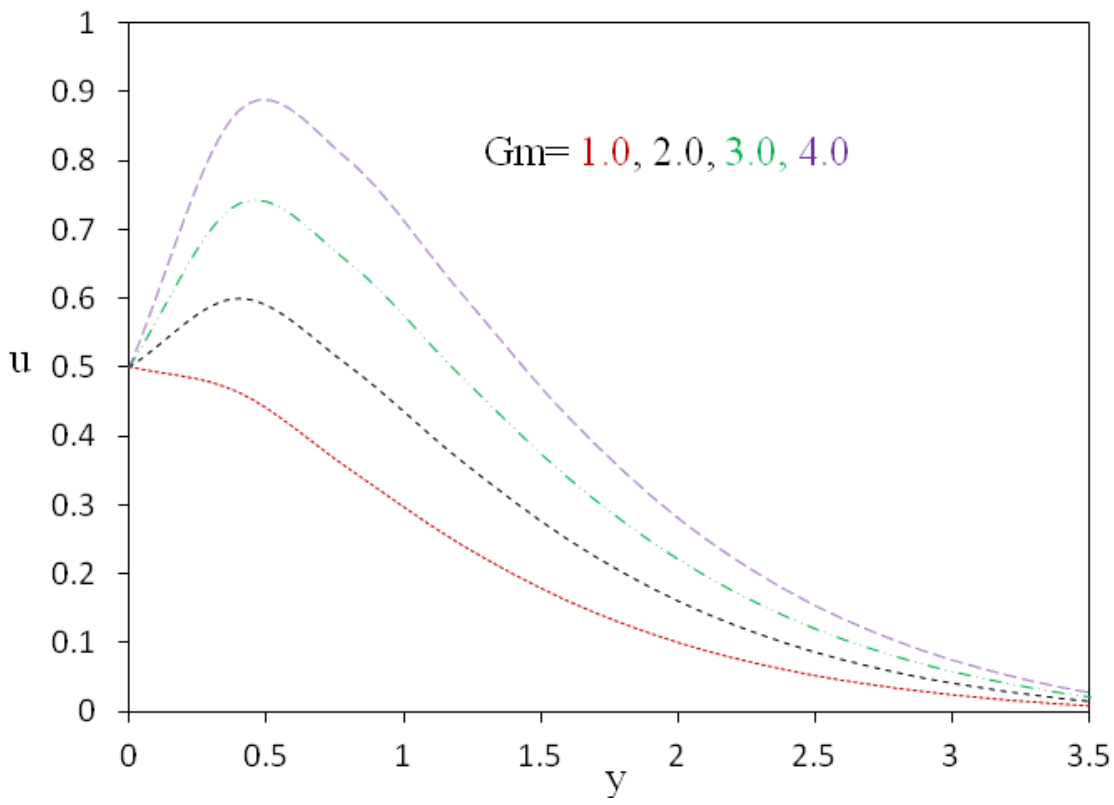

Fig.2. Velocity profiles against $y$ for different values of $G m$ 


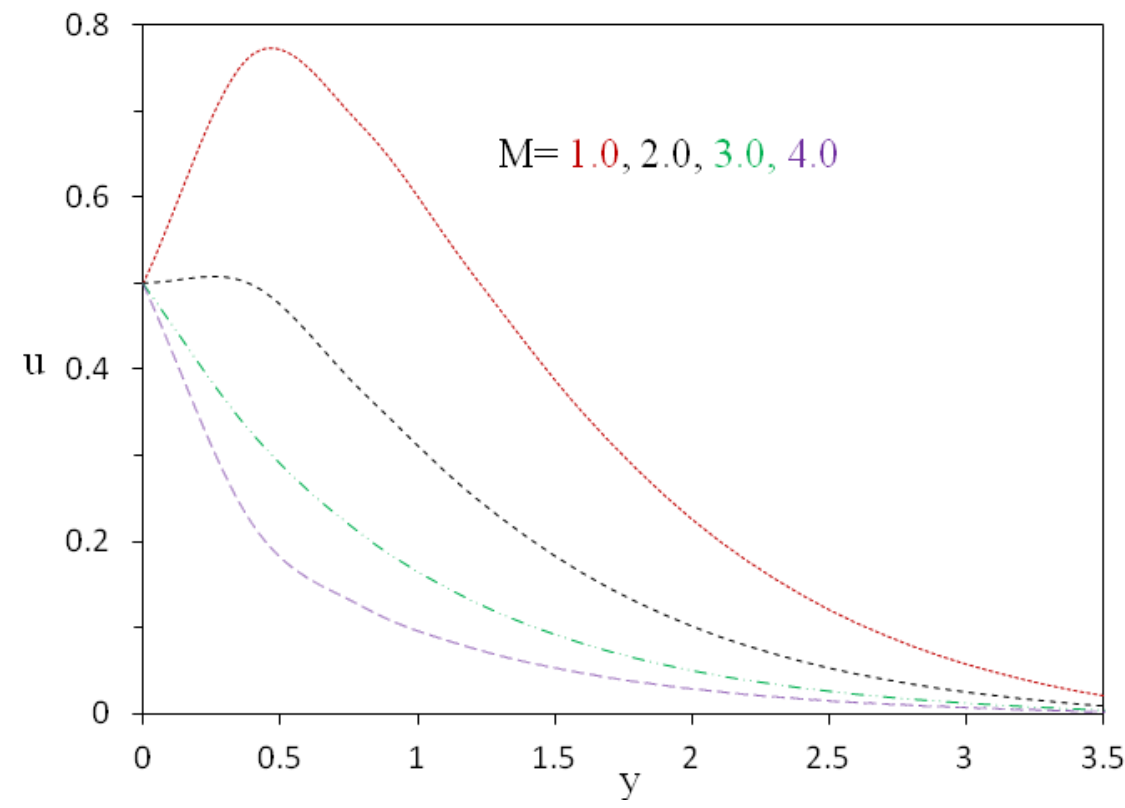

Fig.3. Velocity profiles against $y$ for different values of $M$

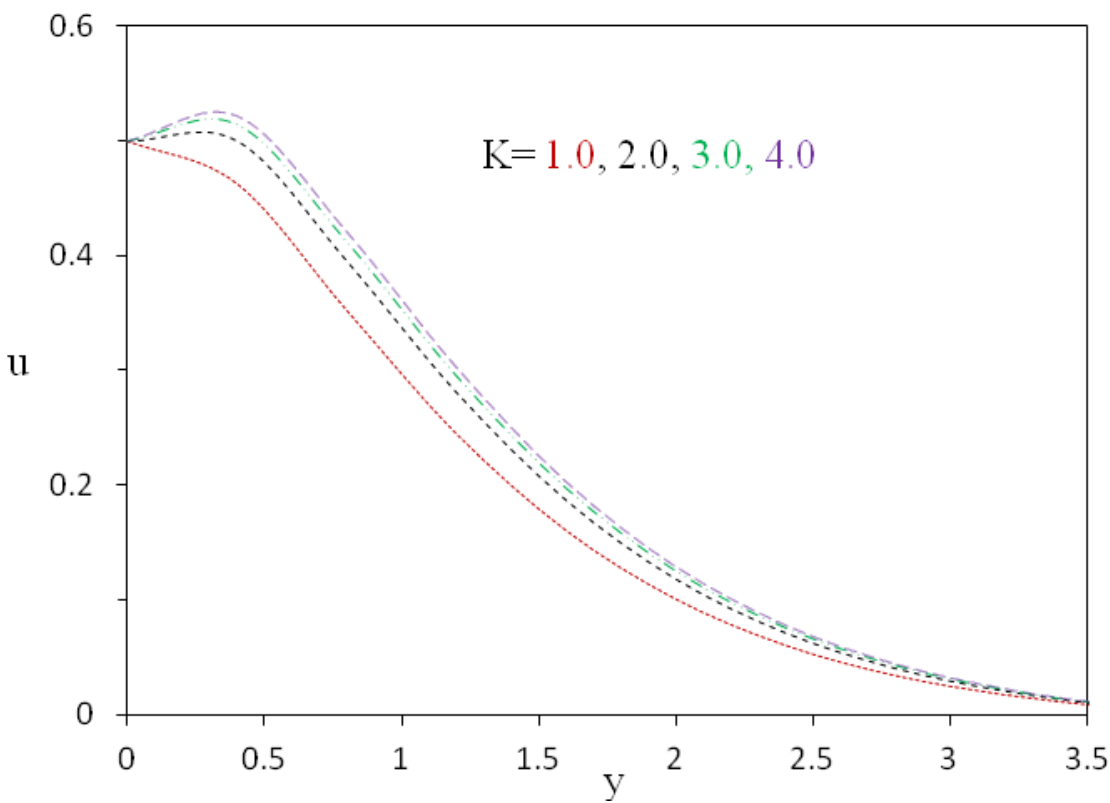

Fig.4. Velocity profiles against $y$ for different values of $K$ 


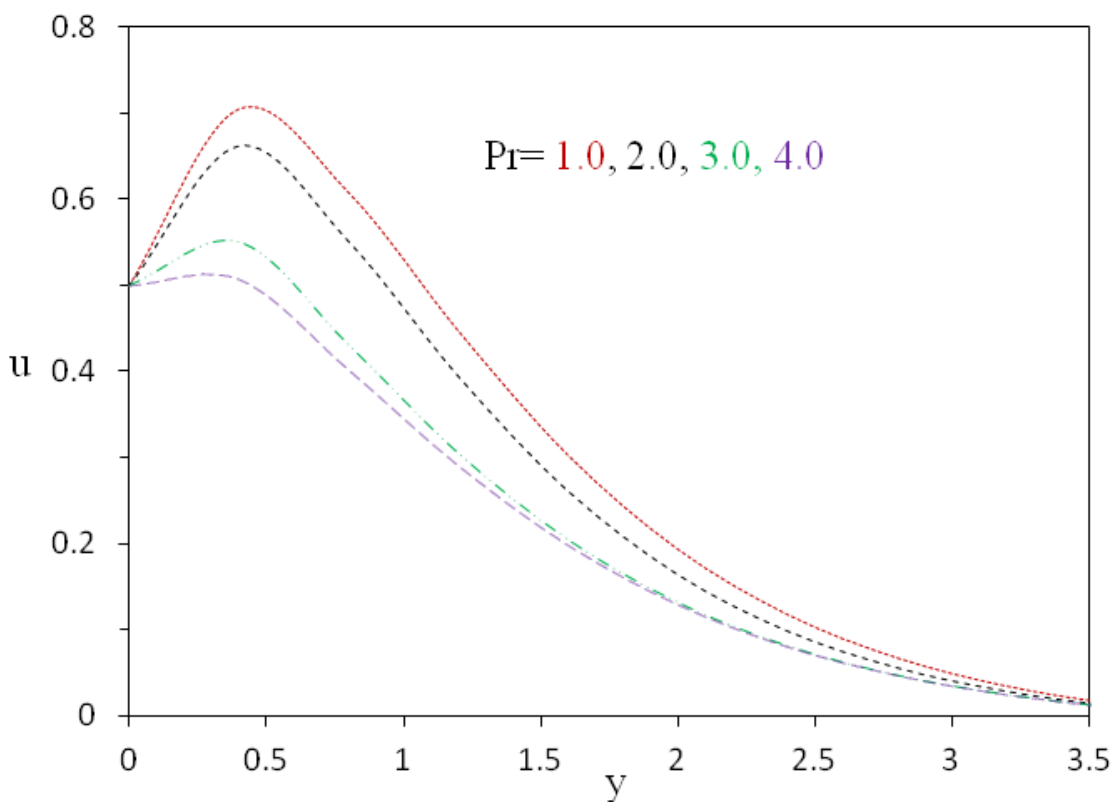

Fig.5. Velocity profiles against $y$ for different values of $\mathrm{Pr}$

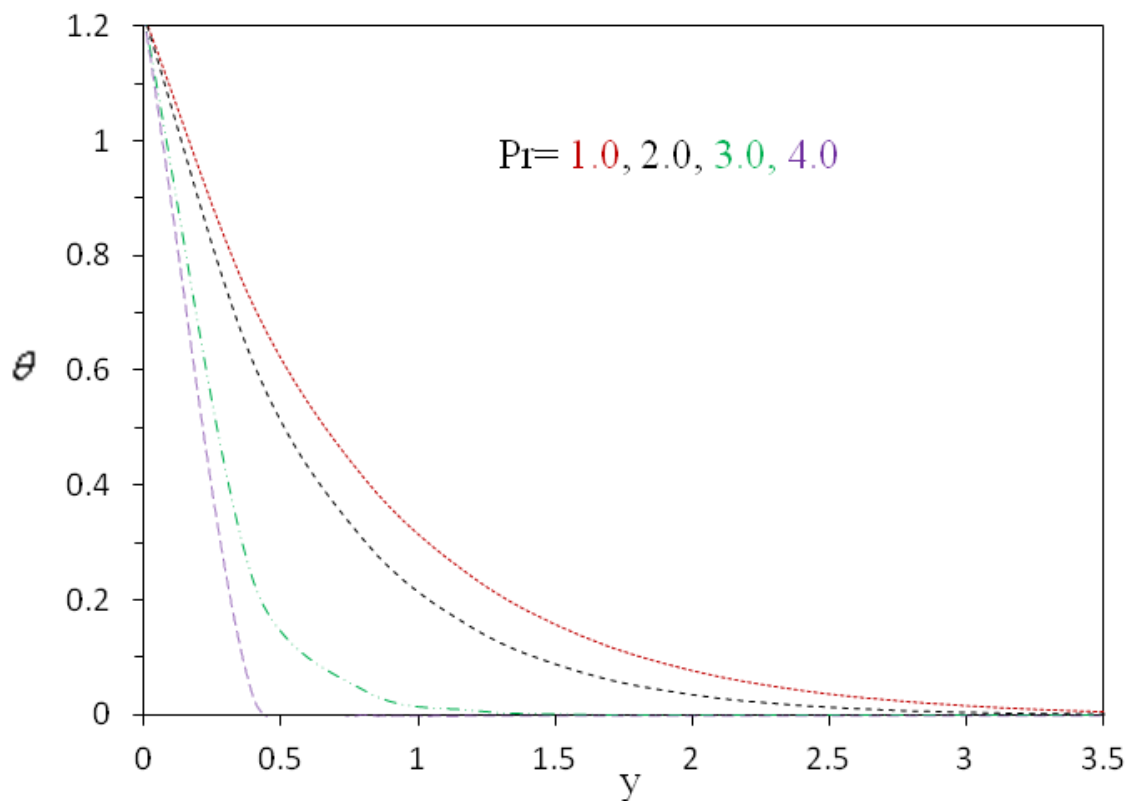

Fig.6.Temperature profiles against $y$ for different values of $\operatorname{Pr} \theta$ 


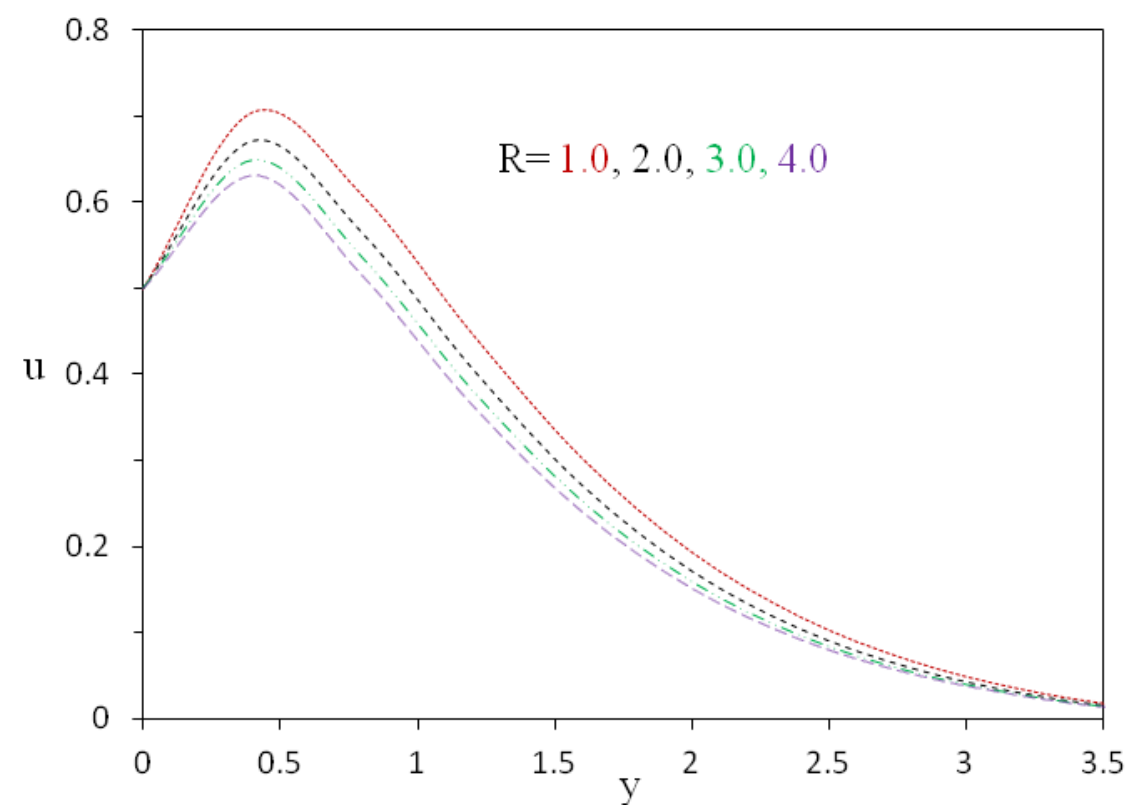

Fig.7. Velocity profiles against $y$ for different values of $R$

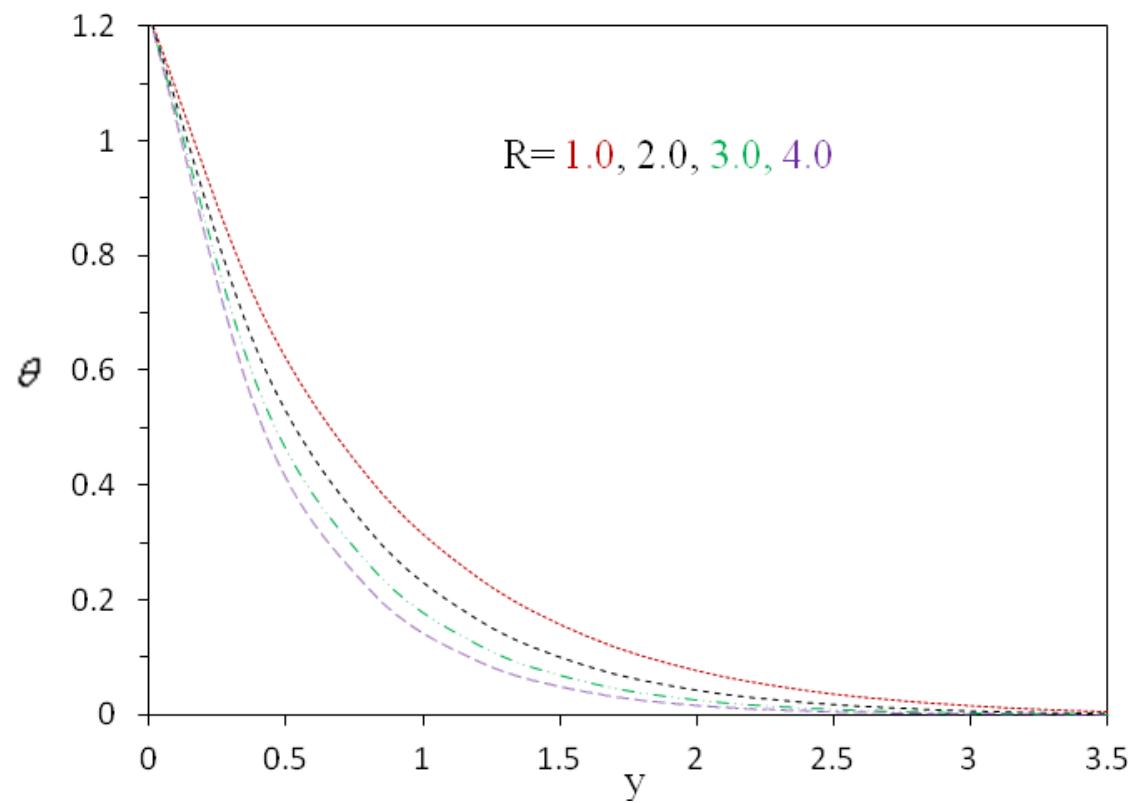

Fig.8.Temperature profiles against $y$ for different values of $R$ 


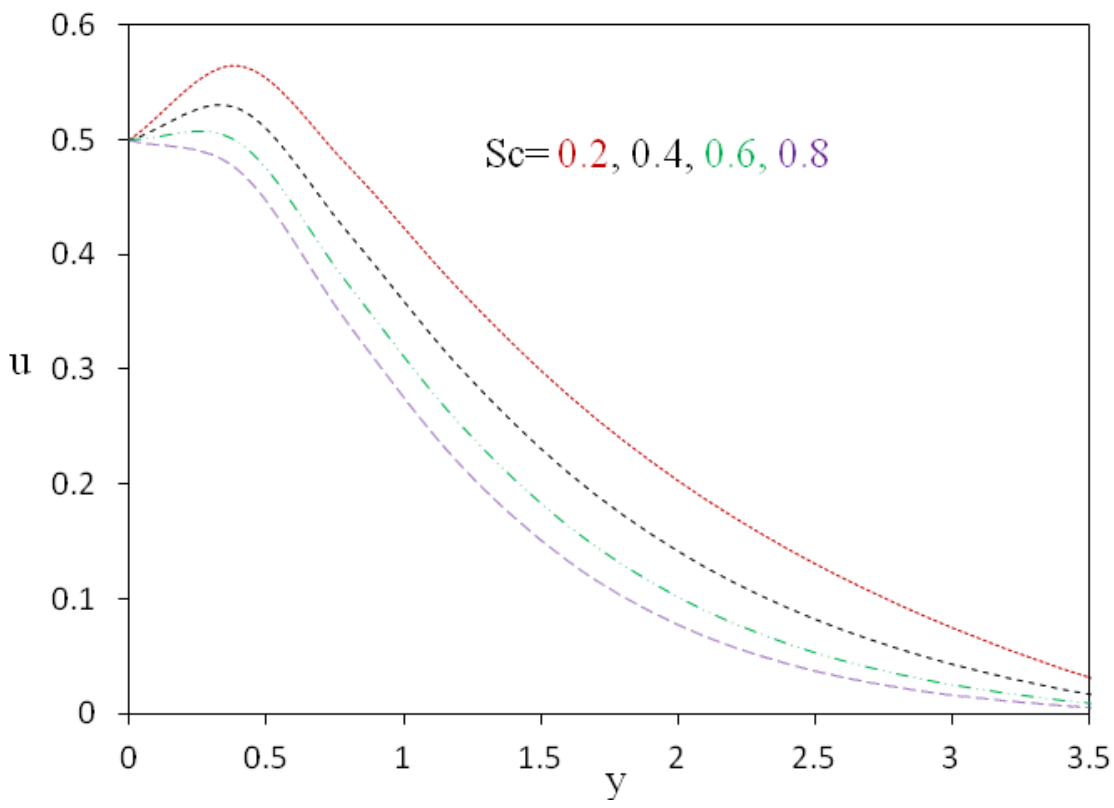

Fig.9. Velocity profiles against $y$ for different values of $S c$

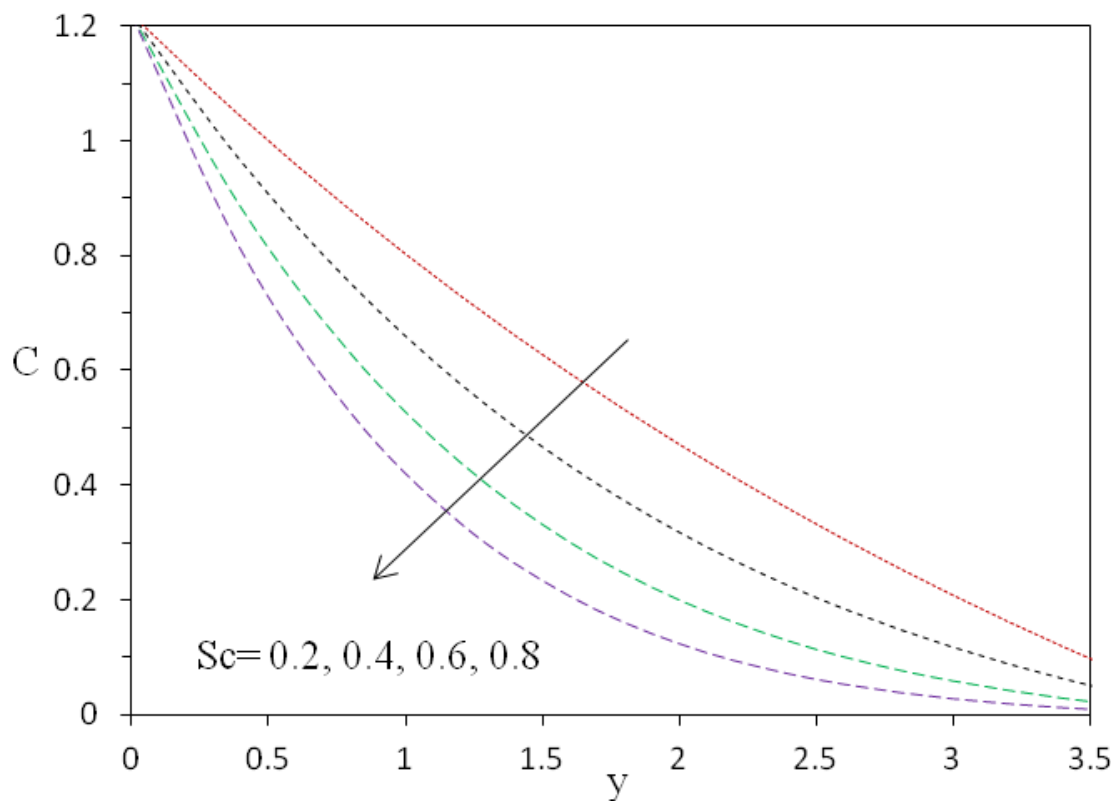

Fig.10. Concentration profiles against $y$ for different values of $S c$ 


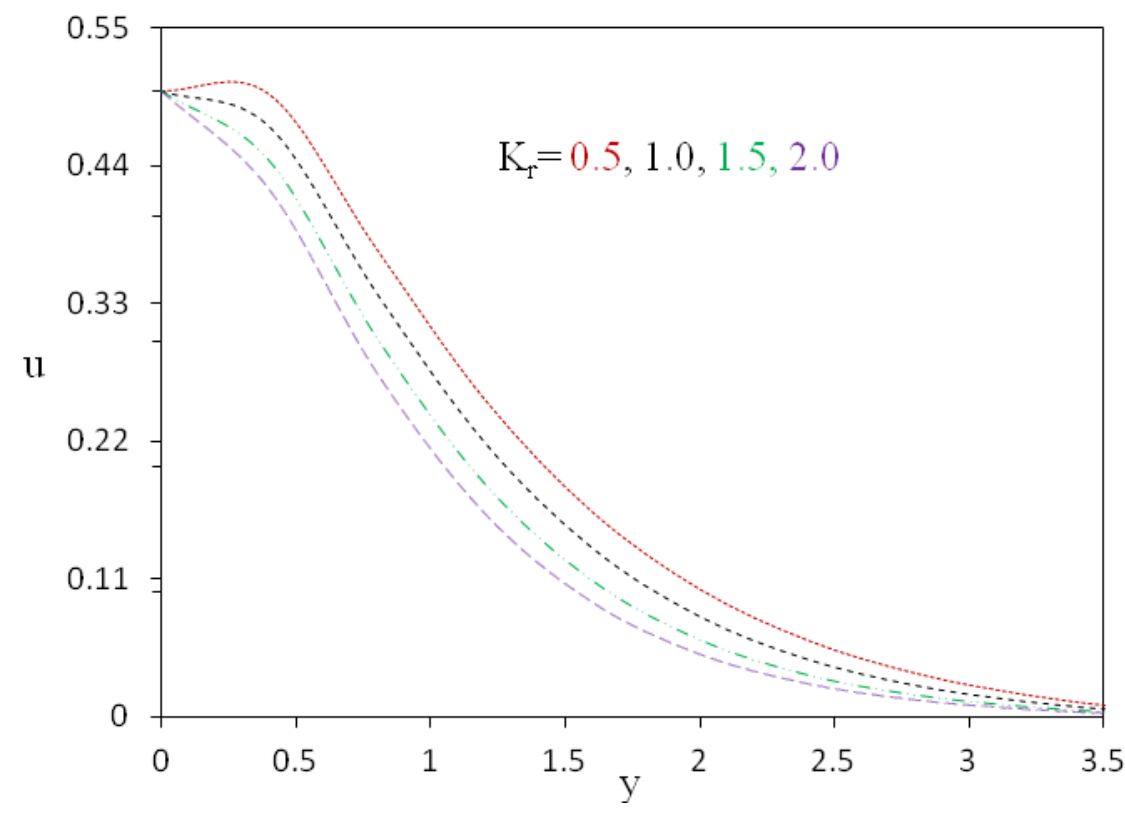

Fig.11. Velocity profiles against $y$ for different values of $K_{r}$

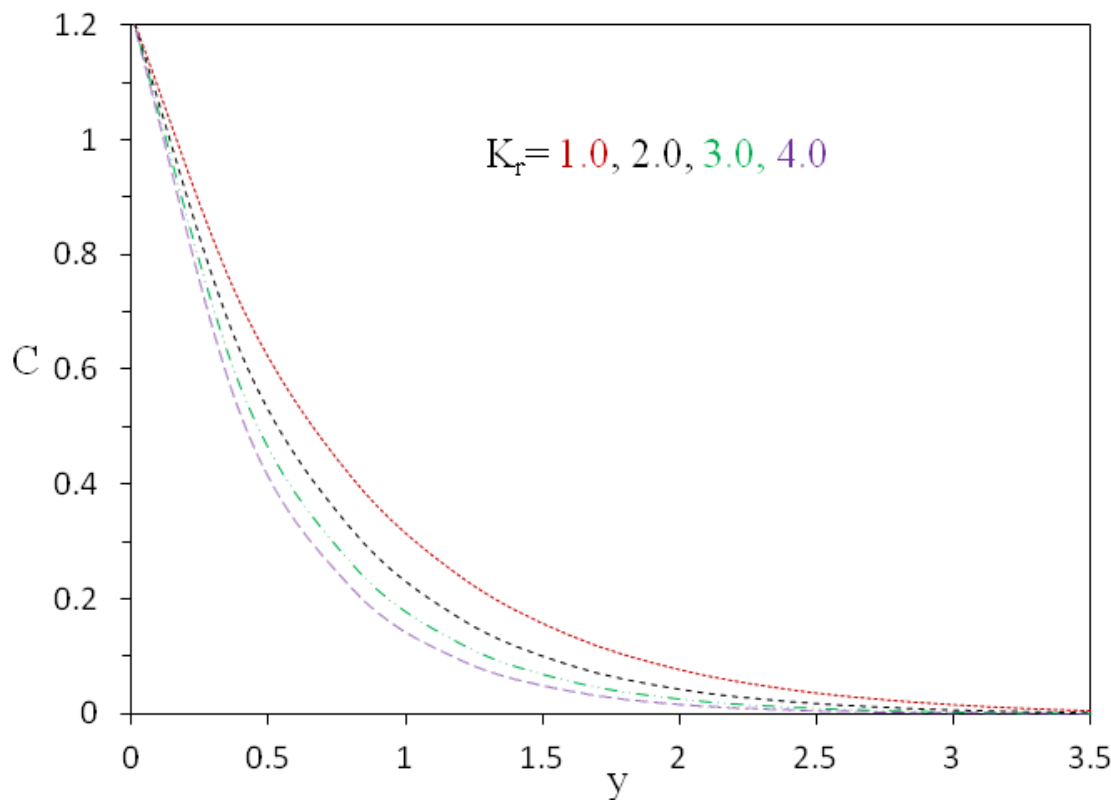

Fig.12.Concentration profiles against $y$ for different values of $K_{r}$ 